\title{
Low-Grade Nasopharyngeal Papillary Adenocarcinoma
}

\author{
Lixin Wang1, Qiaoyun Min1, Jingui Jiang1, Yuyu Shao², Yang Cao² and Daxiang Yan² \\ Department of Pathologyl / Imaging2, Jinhu County People's Hospital, Jiangsu Province, China
}

\begin{abstract}
Low-grade nasopharyngeal papillary adenocarcinoma is a rare tumor, and only a limited number of cases are reported in the literature. The case reported in this study had long-term nasal catarrh with a runny nose and was admitted to the hospital. Computed tomography (CT) examination revealed polypoid mass in the nasopharynx. Pathological examination revealed typical papillary growth pattern of glandular epithelial cells. Immunohistochemical (IHC) staining showed the tumor cells to be diffusely positive for cytokeratin 7 (CK7), vimentin, and thyroid transcription factor 1 (TTF-1). The Ki-67 proliferation index was approximately 1\%. In situ hybridization for latent Ebstein-virus (EBV) injection was negative. The patient did not exhibit recurrence or metastasis of the tumor.
\end{abstract}

Key Words: Nasopharyngeal papillary adenocarcinoma, Clinical features, Pathological diagnosis.

How to cite this article: Wang L, Min Q, Jiang J, Shao Y, Cao Y, Yan D. Low-grade nasopharyngeal papillary adenocarcinoma. J Coll Physicians Surg Pak 2019; 29(11):1114-5.

\section{INTRODUCTION}

Nasopharyngeal cancer (NPC) is common in Southeast Asian countries, especially in the southern part of China. ${ }^{1}$ It originates from the nasopharyngeal epithelial lining and has a variety of pathological subtypes. A majority of the nasopharyngeal carcinomas include keratinizing or non-keratinizing squamous cell carcinoma. ${ }^{2}$ Other pathological types of nasopharyngeal carcinoma include adenocarcinoma, lymphoma, sarcoma, and minor salivary gland tumors. These account for $<5 \%$ of all the nasopharyngeal carcinomas. ${ }^{3}$ The nasopharyngeal papillary adenocarcinoma (NPAC) is a rare, low-grade, malignant tumor, constituting $<1 \%$ of all nasopharyngeal malignancies. It was first reported by Wenig et al. in $1988 .{ }^{4}$

Herein, we report a case of a 77-year female with primary low-grade nasopharyngeal papillary adeno-carcinoma (LGNPPA), who was treated in our hospital. The clinicopathological features of the disease were analysed in a retrospective manner in order to understand the tumor etiology.

\section{CASE REPORT}

A 77-year woman had a history of left nasal catarrh and mild post-nasal drips for 3 years. Head and neck computed tomography (CT) scan revealed left nasopharyngeal lesion (Figure 1). However, no any other related medical history was detected, except for the irregular displacement of the nasal septum. The cervical lymph nodes

Correspondence to: Dr. Jingui Jiang, Department of Pathology,

Jinhu County People's Hospital, Huaian City, Jiangsu

Province, 211600, China

E-mail:15189521184@163.com

Received: December 05, 2018; Revised: March 07, 2019;

Accepted: March 28, 2019 were not enlarged, and the physical examination results were not significant. Any other positive lesions were not detected on CT scan and ultrasound examination of the thyroid gland. An excisional biopsy was performed under local anesthesia using a biopsy fiberscope.

Microscopically, the tumor displayed invasive tumor, with no capsule. The tumor was composed of glandular structures lined by mono- to multi-layered a cuboidal to columnar epithelium. The glandular epithelium was extending into the lumen in papillary configuration with fibrovascular septae in the centre of papillary processes (Figure 2). The tumor cells showed mildly eosinophilic and moderate amount of cytoplasm. The nuclei were round and oval, and the stroma had spindle shaped nuclei. The tumor cell nucle showed mild-to-moderate heterogeneity, mild hyperchromasie and inconspicuous nuclei. The nuclear chromatin was fine to slightly coarse, evenly distributed, and showed vacuoles. The mitoses were rare.

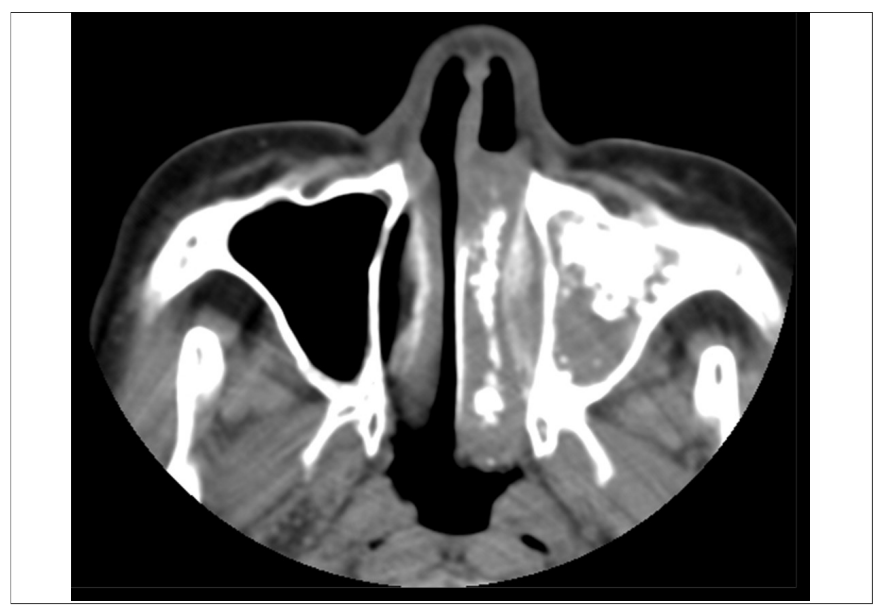

Figure 1: Pedunculated mass in the nasopharynx. 


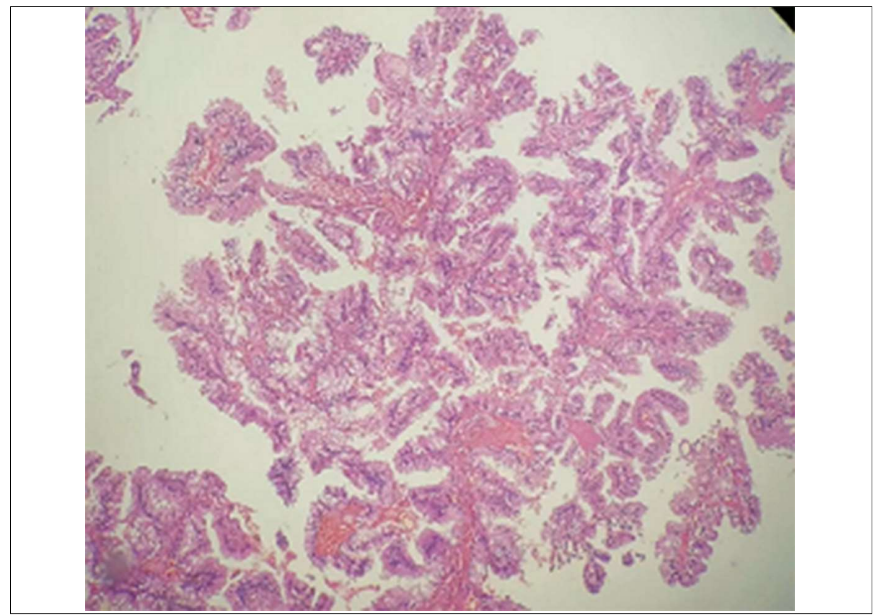

Figure 2: Tumor cells grow as papillary structures lined by cuboidal to columnar cells with low-grade appearance.

IHC revealed cytokeratin 7 (CK7), vimentin, and thyroid transcription factor 1 (TTF-1) positivity in tumor cells, Ki-67 was positive, in around $1 \%$ of the tumor cells. A final diagnosis of primary NPAC was made. In situ bybridization for latent EBV injection was negative.

\section{DISCUSSION}

Primary NPAC is a rare type of tumor, accounting for $0.48 \%$ of all types of NPC. ${ }^{5}$ The NPAC can occur at any age irrespective of the gender. The typical symptoms at presentation include nasal catarrh, while other symptoms include a runny nose, nasal hemorrhage, otitis media, and hearing loss. Typically, the tumor involves the superior, lateral, and posterior walls of the nasopharynx, and is characterised by phytic papillary or nodular swelling. IHC staining shows positivity for CK7 vimentin, and TTF-1. The review of NPAC in Chinese and English literature found that a majority of the tumors were TTF-1 positive. Studies published in English did not demonstrate any TTF-1 negative cases, while only one case of NPAC in Chinese literature was positive for TTF-1.6 Intriguingly, NPAC originates from the mucosal surface lining epithelium. ${ }^{1}$ Microscopic examination of this case demonstrated that the tumor was originating from the lining epithelium of the mucosal surface, which further attests that it originates from the mucosal lining epithelium. The cause of this tumor is not yet clarified, and the current study did not detect any EBV and human papillomavirus (HPV) infections. 7,8

The differential diagnosis of the tumor includes metastatic and ectopic thyroid papillary carcinoma, polymorphous low-grade adenocarcinoma of the salivary glands, papillary intestinal adenocarcinoma, polymorphic adenoma, and pulmonary metastatic papillary adenocarcinoma.

Careful microscopic examination coupled with $\mathrm{IHC}$ and the imaging findings can help arrive at the correct diagnosis.

\section{CONFLICT OF INTEREST:}

Authors declared no conflict of interest.

\section{AUTHORS' CONTRIBUTION:}

LW: Drafted this manucript.

QM, YS, YC, DY: Analysed the data.

$\mathrm{JJ}$ : Supervised and modified the article.

\section{REFERENCES}

1. Wei WI, Sham JS. Nasopharyngeal carcinoma. Lancet 2005; 365:2041-54.

2. Kuo T, Tsang NM. Salivary gland type nasopharyngeal carcinoma: A histologic, immunohistochemical, and EpsteinBarr virus study of 15 cases including a psammomatous mucoepidermoid carcinoma. Am J Surg Pathol 2001; 25:80-6.

3. Schramm VL Jr, Imola MJ. Management of nasopharyngeal salivary gland malignancy. Laryngoscope 2001; 111:1533-44.

4. Wenig BM, Hyams VJ, Heffner DK. Nasopharyngeal papillary adenocarcinoma. A clinicopathologic study of a low-grade carcinoma. Am J Surg Pathol 1988; 12:946-53.

5. Tang Q, Hu QY, Piao YF. Clinical analysis of twentythree nasopharyngeal adenocarcinoma patients. Chin J Cancer Prev Treat 2009; 14:1669-72.

6. Meihui Long. Primary nasal septal papillary adenocarcinoma of case: A literature review. J Otolaryngol Ophthalmol Shandong Uni 2015; 4:86-7.

7. Ohe C, Sakaida N, Tadokoro C, Fukui H, Asako M, Tomoda K, et al. Thyroid-like low-grade nasopharyngeal papillary adenocarcinoma: Report of two cases. Pathol Int 2010; 60:107-11.

8. Minhua Li, Jiangguo Wei, Xiaofei Yao, Cheng Wang. Clinicopathological features of low-grade thyroid-like nasopharyngeal papillary adenocarcinoma. Cancer Res Treat 2017; 49:213-8. 